\title{
Comparing Nasal Cavity Radiotherapy Using Electron, Photon, Proton and Photon-Electron Beams
}

\author{
Navid Khaledi' ${ }^{\text {, Foad Goli Ahmadabad }}{ }^{2}$, Susan Ebam ${ }^{3}$, Roghiye Bodaghi Hosseinabadi4 \\ ${ }^{1}$ Department of Radiation Oncology, Imam Hossein Hospital, Shahid Beheshti University of Medical Science, Tehran, Iran \\ ${ }^{2}$ School of Medicine, Jiroft University of Medical Sciences, Jiroft, Iran \\ ${ }^{3}$ Department of Environmental Health engineering, Jiroft university of Medical Sciences, Jiroft, Iran \\ ${ }^{4}$ Department of Environmental Health, Khoy University of Medical Science, Khoy, Iran \\ Email: *khaledi@sbmu.ac.ir
}

How to cite this paper: Khaledi, N., Ahmadabad, F.G., Ebam, S. and Hosseinabadi, R.B. (2018) Comparing Nasal Cavity Radiotherapy Using Electron, Photon, Proton and Photon-Electron Beams. Journal of Cancer Therapy, 9, 255-261.

https://doi.org/10.4236/jct.2018.93022

Received: October 19, 2017

Accepted: March 11, 2018

Published: March 14, 2018

Copyright (c) 2018 by authors and Scientific Research Publishing Inc. This work is licensed under the Creative Commons Attribution International License (CC BY 4.0).

http://creativecommons.org/licenses/by/4.0/

\begin{abstract}
Aim: Electron, photon or proton beams are used in radiotherapy for cancer treatment while each one may be used depending on depth and the location of tumor and normal tissues around the treatment target as well as economic issues. Materials and Methods: In this research, dose distribution by proton was measured by film dosimetry in nasal cavity Plexiglas phantom and Monte Carlo simulation. Then the DVH of treatment target and the posterior of treatment target of different beams were compared. The energies of electron, photon and proton were $9 \mathrm{MeV}, 6 \mathrm{MV}$, and maximum $65 \mathrm{MeV}$, respectively. Due to a depth of $3.5 \mathrm{~cm}$ of CTV (Clinical Target Volume), Modulation Range was between $0-3.5 \mathrm{~cm}$ and SOBP (Spread-out Bragg Peak) was between 0 - $65 \mathrm{MeV}$. Results: Comparing the obtained DVH values, 95\% dose coverage of target volume for electron, photon, proton and Photon-Electron beams were $88 \%, 98 \%, 98 \%$, and $95 \%$, respectively. However, doses above $40 \%$ that reached outside the target were $50 \%, 82 \%, 5 \%$, and $44 \%$, respectively. Conclusions: The results demonstrate the superiority of proton therapy in nasal cancer due to its better target volume coverage and the less amount of the dose reaching outside the target that is because of dose discharge in a small area and significant dose fall-off after Bragg peak.
\end{abstract}

\section{Keywords}

Electron, Photon, Proton, Mixed Beam, Monte Carlo, Film Dosimetry, Nasal Cavity Cancer

\section{Introduction}

Depending on the position and depth of a tumor, electron, photon, mixed elec- 
tron-photon, or proton beams may be used in radiation therapy. For shallow-seated lesions, in this study nasal-cavity lesions, the most common method for treatment is using electron beam, because of its low penetration depth and therefore sparing the beyond organs from receiving the radiation dose.

Some investigation has been performed about comparison of mixed electron-photon beam and conventional methods [1] [2] [3]. These studies showed that the mixed beam has some advantages over electron or photon beams. For example, the electron beam is more sensitive to small fields [4] [5] than mixed or photon beams.

Xiong et al. conducted a research to optimize dose distribution of photon and electron combination in breast [6]. They compared three techniques that one of these techniques was the combination of photon IMRT and electron IMRT. Their research showed that a combination of photons and electrons reduces the lack of uniformity in the target significantly. In another study, a comparison between combination of IMRT and conformal electron treatment method along with a bolus for parotid and chest areas had been made in a polyethylene phantom by film dosimetry with a $4 \% / 2 \mathrm{~mm}$ criteria gamma index [7]. They used electron energies of 16 and $20 \mathrm{MeV}$. Consequently, in a combination of 2:1 of electron: photon mixture, a more uniform dose coverage than the pure electron in the chest and parotid area was observed. In addition, coincidence between the pure electron and the mixed field for the parotid and chest was $95.9 \%-98.8 \%$.

In another investigation, a comparison was done between Helical Tomotherapy and mixed beam for Glioblastoma Multiforme (GBM) brain tumors [8]. In here, 20 plans were compared for GBM. Both types of plans in terms of homogeneity index, conformity index, and the dose reached to healthy tissues were compared that the results were similar for the mixed beam and Helical Tomotherapy. Only when a normal tissue was very close to the therapeutic target, the Helical Tomotherapy was able to do a better protection on the normal tissue.

A series of studies have been done on the advantages of proton beam in treatment of nasal, para-nasal and sinus lesions [9]-[13]. The proton therapy (PT) delivered a lower mean dose to desired normal tissues in comparison with the IMRT [10]. In addition, the local control for the PT was better than conventional methods after over 3 years and it showed significantly higher disease-free survival at 5 years [11].

There is no study regarding the comparison of the mentioned different beams. Therefore, because of novelty of mixed electron-photon beam in recent researches [2] [14] and PT in modern radiotherapy centers, the present study can be useful for comparison of such beams. By this way, a Monte Carlo (MC) simulation of dose distribution in a Perspex nasal phantom by PT has been compared with dose distribution by MC calculation and film dosimetry of electron, photon, and mixed beam.

\section{Materials and Methods}

Dose distributions (Isodoses) measurement in a heterogeneous region was per- 
formed by a high dose range (EDR2) Kodak (Rochester, New York) film in a Plexiglas Phantom. The Perspex phantom was used for dosimetry because of its density $\left(1.06 \mathrm{gr} / \mathrm{cm}^{3}\right)$ that is close to soft tissue. Film dosimetry in this Plexiglas phantom allows us to estimate the dose distribution of the mixed photons and electrons beam as well as the pure photon or electron inside the phantom, especially in the heterogeneous areas (nasal and sinus). The Plexiglas phantom was built of twenty slices with thickness of $1 \mathrm{~cm}$.

Because of the specialty of the treatment planning systems algorithms to calculate the dose distribution in material for cases merely photon or merely electron, the MC codes can be a good option for calculation of dose distribution.

A Varian 2300 CD linear accelerator was used in this study as a source of the photon or electron beams. The dose rate in this linac is $300 \mathrm{MU} / \mathrm{Min}$ in normal mode that can be increased to $1000 \mathrm{MU} / \mathrm{Min}$. The distance of applicator tray to source is $63 \mathrm{~cm}$. The length of applicator is also $32 \mathrm{~cm}$; it means the applicator end distance to Isocenter is $5 \mathrm{~cm}$.

A clinical tumor volume (CTV) with dimensions $3 \times 3.5 \mathrm{~cm}^{2}$ was considered in the nasal cavity of the phantom (Figure 1). The irradiation was performed by placing each film among the middle slice of phantom (ten slices from each side) and the SSD was $100 \mathrm{~cm}$. Film dosimetry was conducted for $9 \mathrm{MeV}$ electron beam and photon beam of $6 \mathrm{MV}$ and one mode of the mixed beam (20/80 ratio of photon to electron mixture).

In order to calibrate the optical density (OD) and to obtain the film dosimetry calibration curve, each calibration films were placed in water-equivalent sheets of RW3 (PTW, Freiburg) with density of $1.05 \mathrm{gr} / \mathrm{cm}^{3}$. The thickness of each sheets were $1 \mathrm{~cm}$. In this case, films were placed perpendicular to the beam's central axis.

After designing and introducing geometrical features of linac, including collimator, primary and secondary scattering foil and applicator as well as lead and steel shielding and also water phantom and Plexiglas phantom in AutoCAD software, the MC simulation was started. The applied MC code was MCNPX v2.6 [15]. The library used to transport photon was MCLIB04, and EL03 was also used for electron. In addition, the cut-off energies for electron and photon were chosen 0.5 and $0.03 \mathrm{MeV}$, respectively. Importance of all materials (cells) in the simulation was selected equal to unit (IMP $=1)$.

To obtain the beam quality and distribution of the electron beam exited from Bending Magnet in MC, the PDD and profile in a water phantom should be measured. As a result, by changing the energy FWHM, peak energy, spatial FWHM, and its angular distribution, the measured and simulated PDDs and profiles matched on each other. The most important parameters of conformity of measurement and simulation electron PDDs are the depth of $50 \%$ dose $\left(\mathrm{R}_{50}\right)$ and the practical range $\left(\mathrm{R}_{\mathrm{P}}\right)$.

For isodose simulation of proton beam, two wax wedges placed at the path of the protons as compensators, compensating the shape of nose (Figure 1). The Spread-out Bragg Peak (SOBP) was between $0-65 \mathrm{MeV}$ with a modulation 


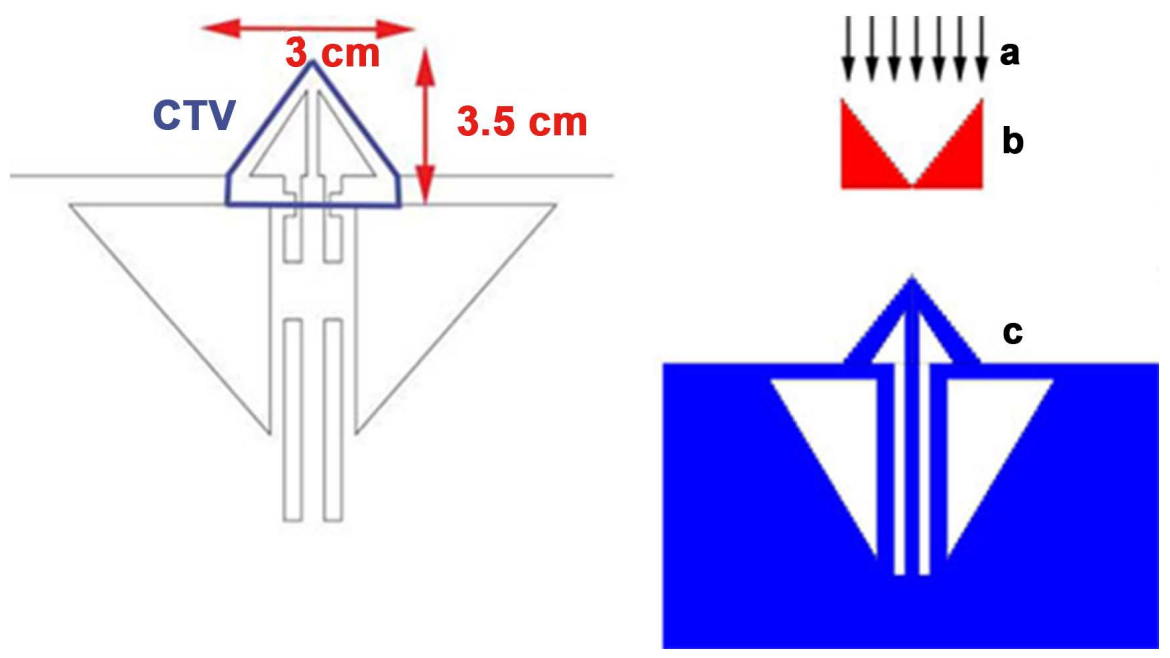

Figure 1. Right-side: (a) proton beam, (b) wax compensator, (c) nasal Plexiglas phantom, and the left-side, the nasal phantom design in measurement and MC simulation.

range of $0-3.5 \mathrm{~cm}$. Moreover, the proton source distribution was a $3.2 \times 3.2 \mathrm{~cm}^{2}$ square $(0.2 \mathrm{~cm}$ wider than CTV size for penumbra consideration), placed $30 \mathrm{~cm}$ above the nasal phantom.

\section{Results}

Figure 2 shows the obtained calibration curve for film dosimetry used in the Verisoft software. This calibration curve used for all measured dose distributions in film dosimetry.

The area of nasal region and Ethmoid sinus were divided to $2 \times 2 \mathrm{~cm}^{2}$ segments, by calculation of the delivered dose to each of these segments dose-surface-histogram was obtained. By extracting the DSH data, the coverage of CTV by isodose of $95 \%$, as well as, the delivered doses higher than $40 \%$ of prescribed dose to the normal tissues outside of the CTV showed in Table 1. As can be seen from this table, the best coverage of CTV is for $6 \mathrm{MV}$ photon and 0 - 65 $\mathrm{MeV}$ protons, but, the maximum doses to the normal tissues irradiated by $6 \mathrm{MV}$ photon (82\%).

By comparing the results in Table 1, it is clear that the balance between CTV coverage and dose delivery to normal tissues belongs to proton beam (5\%). Next, the mixed beam with $44 \%$ dose to normal tissues shows the second good result.

The isodose color-wash for nasal cavity in proton therapy mode is illustrated in Figure 3. In this figure the good coverage of CTV and low isodose levels in the outside of the CTV, obviously visible. In here, the coverage of $100 \%$ isodose in nasal cavity and $10 \%$ isodose reached to OARs are more tangible than other techniques.

\section{Discussion}

Due to the sensitivity of electron beam to heterogeneity and the shape of irradiated medium, the dose coverage between the nasal holes, with 55\% isodose, 


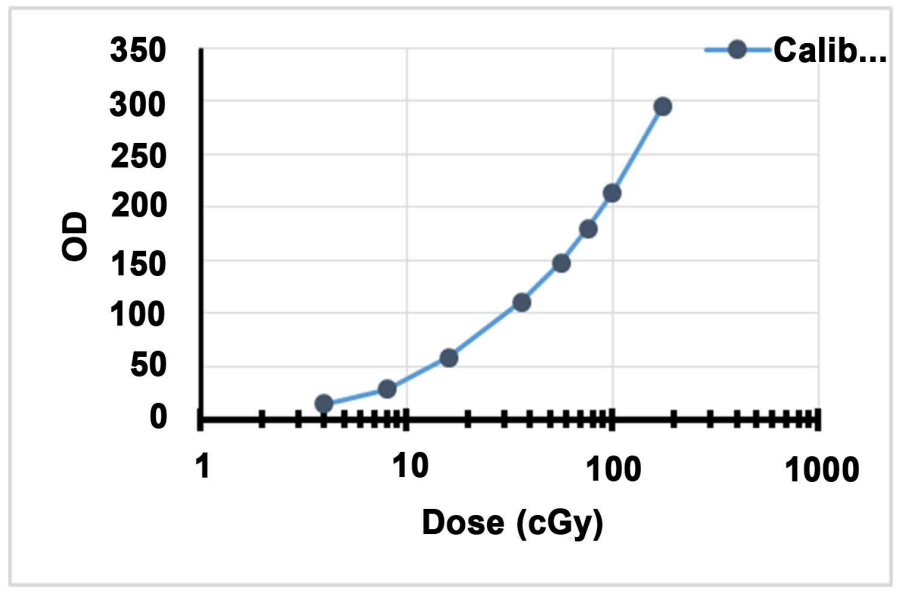

Figure 2. The calibration curve obtained from irradiating different doses to the film.

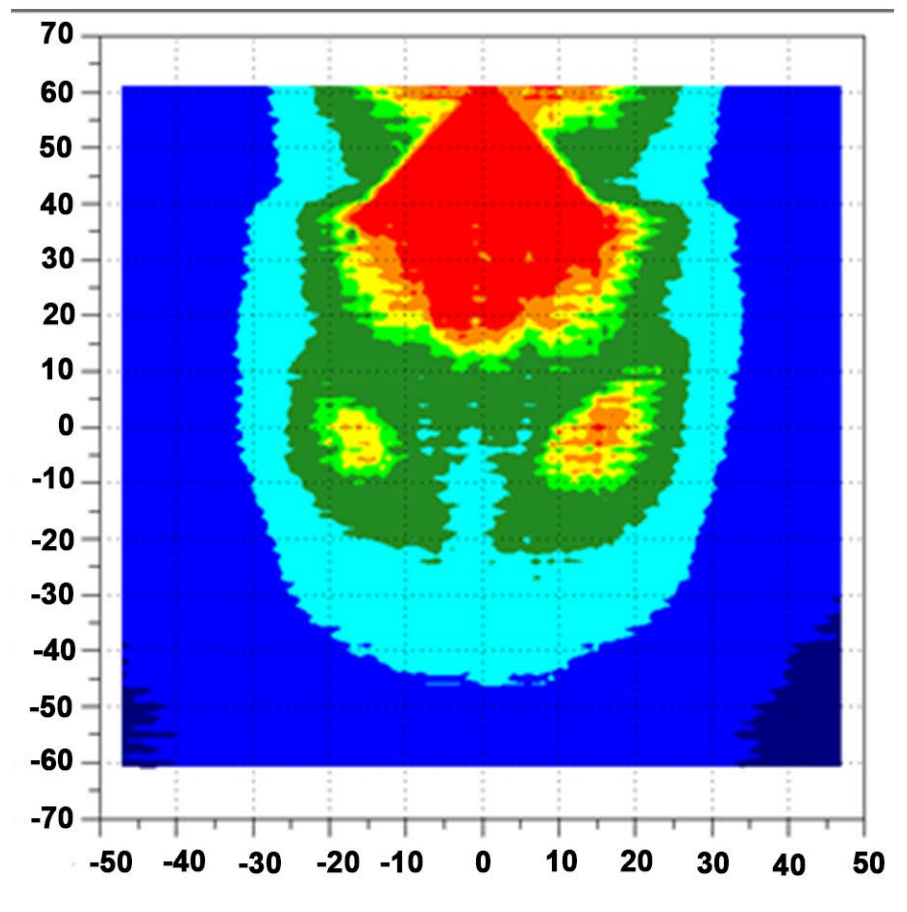

Figure 3. The simulated dose distribution of proton beam in nasal phantom.

Table 1. The CTV coverage of $95 \%$ isodose and the delivered doses above $40 \%$ to the Normal tissues beyond the CTV.

\begin{tabular}{ccc}
\hline Beam type & CTV coverage (\%) & Delivered dose to normal tissues (\%) \\
\hline $9 \mathrm{MeV}$ electron beam & 88 & 50 \\
$6 \mathrm{MV}$ photon beam & 98 & 82 \\
Mixed beam & 95 & 44 \\
Proton beam & 98 & 5 \\
\hline
\end{tabular}


was very poor. For the photon beam, due to the low gradient of dose fall-off, nevertheless the good coverage of CTV area, the high level dose was present in the outside of the CTV.

However, the sparing of outside of the treatment target, in the mixed beam mode was better than both of electron and photon beams. It showed a better nasal cavity and nasal septum coverage in comparison with the electron beam, as well as better OAR sparing compared with photons. In the proton state, both coverage of nasal cavity and nasal septum was far better than other techniques. In addition, the homogeneity index in the CTV for PT was 11\%, 18\%, 31\% better than, mixed beam, photons, and electrons, respectively.

\section{Conclusion}

This study showed that the excellent coverage of treatment target and sparing the normal tissues by proton beam is in comparison with the other methods. The low amount of doses outside of the target, was very considerable. After this beam, the mixed electron-photon beam had the next stage between the investigated beams. This study, parallel with literature, demonstrate the significant capability of PT for cancer treatment, especially in the nasal case that the high amount of delivered dose arisen from the SOBP tail in the single field is not matter. But the economic issues in application of proton beam could not be neglected, because this type of treatment is very expensive than other mentioned methods.

\section{References}

[1] Blasi, O., Fontenot, J.D., Fields, R.S., Gibbons, J.P. and Hogstrom, K.R. (2011) Preliminary Comparison of Helical Tomotherapy and Mixed Beams of Unmodulated Electrons and Intensity Modulated Radiation Therapy for Treating Superficial Cancers of the Parotid Gland and Nasal Cavity. Radiation Oncology, 6, 178. https://doi.org/10.1186/1748-717X-6-178

[2] Khaledi, N., Arbabi, A., Sardari, D., Mohammadi, M. and Ameri, A. (2015) Simultaneous Production of Mixed Electron-Photon Beam in a Medical LINAC: A Feasibility Study. Physica Medica, 31, 391-397. https://doi.org/10.1016/j.ejmp.2015.02.014

[3] Mu, X., Olofsson, L., Karlsson, M., Sjögren, R. and Zackrisson, B. (2004) Can Photon IMRT Be Improved by Combination with Mixed Electron and Photon Techniques? Acta Oncologica, 43, 727-735. https://doi.org/10.1080/02841860410002761

[4] Khaledi, N., Arbabi, A., Sardari, D., Mahdavi, S.R., Aslian, H., Dabaghi, M. and Sheibani, K. (2013) Monte Carlo Investigation of the Effect of Small Cutouts on Beam Profile Parameters of 12 and $14 \mathrm{MeV}$ Electron Beams. Radiation Measurements, 51-52, 48-54. https://doi.org/10.1016/j.radmeas.2013.01.019

[5] Khaledy, N., Arbabi, A. and Sardari, D. (2011) The Effects of Cutouts on Output, Mean Energy and Percentage Depth Dose of 12 and $14 \mathrm{MeV}$ Electrons. Journal of Medical Physics, 36, 213-219. https://doi.org/10.4103/0971-6203.89970

[6] Xiong, W., Li, J., Chen, L., Price, R., Freedman, G., Ding, M. and Ma, C. (2004) Optimization of Combined Electron and Photon Beams for Breast Cancer. Physics in Medicine \& Biology, 49, 1973-1989. https://doi.org/10.1088/0031-9155/49/10/010 
[7] Kavanaugh, J.A., Hogstrom, K.R., Chu, C., Carver, R.A., Fontenot, J.P. and Henkelmann, G. (2013) Delivery Confirmation of Bolus Electron Conformal Therapy Combined with Intensity Modulated X-Ray Therapy. Medical Physics, 40, 21-28. https://doi.org/10.1118/1.4788657

[8] Koca, T., Basaran, H., Sezen, D., Karaca, S., Ors, Y., Arslan, D. and Aydin, A. (2014) Comparison of Linear Accelerator and Helical Tomotherapy Plans for Glioblastoma Multiforme Patients. Asian Pacific Journal of Cancer Prevention: Asian Pacific Journal of Cancer Prevention, 15, 7811-7816. https://doi.org/10.7314/APJCP.2014.15.18.7811

[9] Fukumitsu, N., Ishikawa, H., Ohnishi, K., Terunuma, T., Mizumoto, M., Numajiri, H. and Sakae, T. (2014) Dose Distribution Resulting from Changes in Aeration of Nasal Cavity or Paranasal Sinus Cancer in the Proton Therapy. Radiotherapy and oncology, 113, 72-76. https://doi.org/10.1016/j.radonc.2014.08.024

[10] McDonald, M.W., Liu, Y., Moore, M.G. and Johnstone, P.A. (2016) Acute Toxicity in Comprehensive Head and Neck Radiation for Nasopharynx and Paranasal Sinus Cancers: Cohort Comparison of 3D Conformal Proton Therapy and Intensity Modulated Radiation Therapy. Radiation Oncology, 11, 32. https://doi.org/10.1186/s13014-016-0600-3

[11] Patel, S.H., Wang, Z., Wong, W.W., Murad, M.H., Buckey, C.R., Mohammed, K. and Schild, S.E. (2014) Charged Particle Therapy versus Photon Therapy for Paranasal Sinus and Nasal Cavity Malignant Diseases: A Systematic Review and Meta-Analysis. The Lancet Oncology, 15, 1027-1038. https://doi.org/10.1016/S1470-2045(14)70268-2

[12] Zenda, S., Akimoto, T., Mizumoto, M., Hayashi, R., Arahira, S., Okumura, T. and Sakurai, H. (2016) Phase II Study of Proton Beam Therapy as a Nonsurgical Approach for Mucosal Melanoma of the Nasal Cavity or Para-Nasal Sinuses. Radiotherapy and Oncology, 118, 267-271. https://doi.org/10.1016/j.radonc.2015.10.025

[13] Zenda, S., Kawashima, M., Arahira, S., Kohno, R., Nishio, T., Tahara, M. and Akimoto, T. (2015) Late Toxicity of Proton Beam Therapy for Patients with the Nasal cavity, Para-Nasal Sinuses, or Involving the Skull Base Malignancy: Importance of Long-Term Follow-Up. International Journal of Clinical Oncology, 20, 447-454. https://doi.org/10.1007/s10147-014-0737-8

[14] Khaledi, N., Sardari, D., Mohammadi, M., Ameri, A. and Reynaert, N. (2018) Dosimetric Evaluation of a Novel Electron-Photon Mixed Beam, Produced by a Medical Linear Accelerator. Journal of Radiotherapy in Practice, 1-13. https://doi.org/10.1017/S1460396917000711

[15] Briesmeister, J.F. (1986) MCNP-A General Monte Carlo Code for Neutron and Photon Transport-Version 3A: Los Alamos National Laboratory Report LA-7396-M. 